\title{
Markers of microvascular complications in insulin dependent diabetes
}

\author{
C J Schultz, R Amin, D B Dunger
}

\section{Annual screening for early markers of microvascular disease during puberty should be encouraged}

$\mathrm{S}$ reening for early markers of microvascular disease is now generally commended from around the age years in children with type diabetes mellitus (TIDM). Annual assessment should include direct fundal examination or fundus photography, monitoring of arterial blood pressure (BP), and measurement of urinary albumin excretion. The basis of all screening programmes is that those most at risk can be correctly identified and that there should be appropriate and effective interventions. The value of retinal screening is beyond doubt as the detection of early preproliferative or proliferative retinopathy may lead to successful intervention with laser therapy, but such events are rare before the age of 18 years. The evidence supporting screening for other markers of microvascular disease, such as microalbuminuria (MA) and hypertension is more contentious and this is the subject of the current review. Symptomatic autonomic or sensory nephropathy is relatively rare in children and adolescents and this will not be discussed further.

\section{WHEN TO SCREEN}

The decision to introduce screening at age 10 years reflects the influence of puberty on the risk for microvascular disease. Several cross sectional studies and at least two large longitudinal studies (the Berlin Retinopathy Study and the Oxford Regional Prospective Study (ORPS)) have shown that microvascular complications are rare before puberty. ${ }^{12}$ Puberty confers a three- to fourfold increase in risk of MA after adjusting for other major risk factors such as diabetes duration, HbAlc, and gender. ${ }^{13}$ Prepubertal duration of diabetes may contribute to the risk of MA, but this only becomes evident after the onset of puberty. ${ }^{1}$ Contrary to historical belief, MA is not rare ${ }^{4}$ and may progress ${ }^{5}$ within the first five years after the diagnosis of TIDM in pubertal subjects. ${ }^{45}$ Puberty also increases the risk of retinopathy by approximately threefold ${ }^{6}$; as a result the median duration of diabetes when retinopathy is detected is significantly shorter in subjects of pubertal age at diagnosis when compared to those where diabetes is diagnosed before puberty. ${ }^{2}$ Preproliferative retinopathy may be detected, using fundus photography, in pubertal subjects as young as 11 years of age and at seven years duration of diabetes.

\section{MICROALBUMINURIA}

The term MA denotes increases in albumin excretion rates outside of the normal range, but too low to register on the normal clinic albusticks. The current consensus definition is that the urinary albumin excretion rate should be more than $20 \mu \mathrm{g} / \mathrm{min}$ and less than $200 \mu \mathrm{g} / \mathrm{min}$ in two of three overnight or 24 hour urines collected within 6-12 months. The predictive value of MA was initially overestimated, but nevertheless in adults approximately $30 \%$ of patients will progress to frank albuminuria (Dipstix positive) with the subsequent risk of renal failure within 10 years. MA is also predictive of cardiovascular disease in adults and will be present in approximately $80 \%$ of patients with proliferative retinopathy.

The timed urine collections needed to estimate albumin excretion rates are often impractical, particularly during childhood, and several studies have indicated that estimation of the albumincreatinine ratio (ACR) on the first urine passed in the morning is an equally valid measure. Storage of urine at $-20^{\circ} \mathrm{C}$ can affect albumin concentrations; storage at $-70^{\circ} \mathrm{C}$ or early measurement of fresh samples is preferable. ${ }^{9}$ Repeated measures are of far greater value than occasional single assessments. Albumin excretion rates show considerable variability from day to day and three urines annually are necessary to confirm the presence and persistence of MA. The risk of nephropathy can probably be detected before the onset of MA by serial measurements of the ACR. ${ }^{10}$

In those who subsequently develop $\mathrm{MA}$, the rate of albumin excretion increases from year to year during childhood; the rate increases quite dramatically during puberty. ${ }^{3}$ After puberty, rates of albumin excretion may decline and MA may be transient in around $50 \%$ of young people with diabetes. However, in those subjects with transient MA, rates of increase during the prepubertal years are similar to those who have persistent MA, and although they may be protected during puberty by lower HbAlc levels, they are probably still genetically at risk of nephropathy. Persistent MA may develop within three years in $20 \%$ of these adolescents with apparently transient MA.

\section{GLYCAEMIC CONTROL}

The risk of developing microvascular complications increases exponentially with increasing HbAlc above the normal level. $^{12}$ In the ORPS cohort approximately $40 \%$ of adolescents with a mean HbAlc (DCCT standardised) of $10.5 \%$ or higher develop MA (transient or persistent) within 12 years from diagnosis of TIDM (fig $1^{11}$ ). For every $1 \%$ increase in $\mathrm{HbAlc}$ there was an associated increase of $36 \%$ in the risk of MA. ${ }^{1}$ The risk of retinopathy also increases exponentially with increasing HbAlc, ${ }^{13}$ and approximately $50 \%$ of those with a mean HbAlc of $10 \%$ or more will develop background retinopathy within 12 years from diagnosis of diabetes. ${ }^{13}$

\section{BLOOD PRESSURE}

Although hypertension is almost always associated with diabetic nephropathy in TIDM, elevations in routine office BP have not consistently been identified before the onset of MA. ${ }^{14}$ However, one small study that measured 24 hour ambulatory BP reported that an increased night to day ratio of arterial BP might precede the development of MA. ${ }^{15}$ In contrast, higher BP levels certainly contribute to the progression of persistent microalbuminuria, ${ }^{5}$ and although BP may not be important in the initiation of diabetic retinopathy, a higher diastolic $\mathrm{BP}$, even within the normal range, may increase the risk of progression of retinal lesions. ${ }^{16}$ The presence of micro- or macroalbuminuria may also modify the effect of BP on the progression of retinopathy. ${ }^{16}$ Thus the identification of hypertension in the clinic population is important, particularly if it is associated with MA.

\section{FAMILY PHENOTYPES AND GENETIC RISK FOR DIABETIC COMPLICATIONS}

The risk of diabetic complications is most likely partly inherited. A family history

Abbreviations: $A C E$, acetylcholinesterase; $A C R$, albumin-creatinine ratio; $B P$, blood pressure; DCCT, Diabetes Control and Complications Trial; MA, microalbuminuria; ORPS, Oxford Regional Prospective Study; TIDM, type 1 diabetes mellitus 


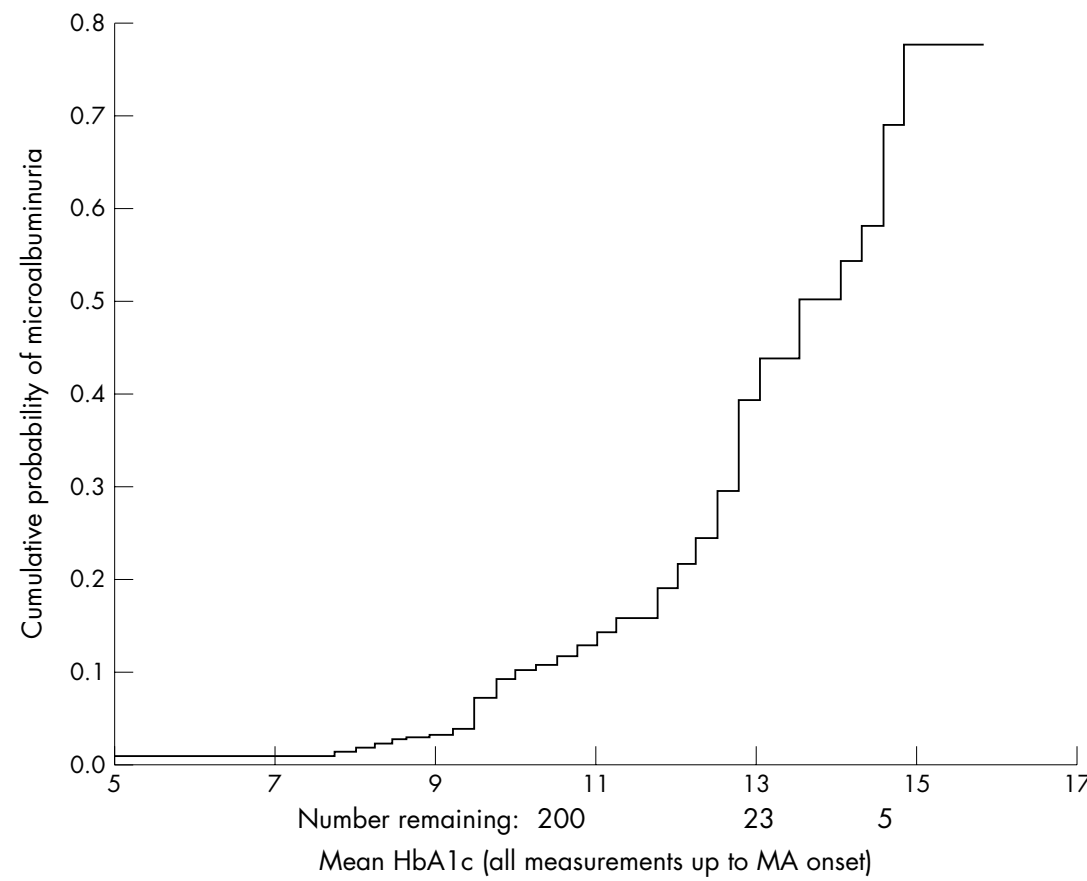

Figure 1 Cumulative probability of MA (transient or persistent) with increasing $\mathrm{HbAlc}$ in the Oxford Regional Prospective Study.

of either diabetic nephropathy or retinopathy is associated with an increased risk of these complications in relatives with TIDM. ${ }^{17}$ Within families a history of parental hypertension is also associated with nephropathy risk in the offspring, ${ }^{18}$ as is a history of type 2 diabetes in the parents. ${ }^{19}$ These familial risk factors have been less often studied in young people with TIDM. However, one study investigated parental factors in 300 young subjects and found that familial hypertension and cardiovascular disease were associated with $\mathrm{MA}$ in the offspring. ${ }^{20}$ Thus family phenotypes may be useful markers of risk of microvascular disease.

Such familial aggregation of complications risk would indicate that genetic factors are important, but as yet, no strong genetic marker for diabetic complications has been identified. The strongest associations have been between nephropathy and the Apo-E polymorphism which may influence lipoprotein levels, and the acetylcholinesterase (ACE) genotype which is known to be related to ACE expression and response to treatment with ACE inhibitors. Currently there are a number of large international collaborations, which hopefully will identify other genetic risk markers. ${ }^{21}$

\section{ENDOCRINE AND GENDER DIFFERENCES}

The observations that puberty is the watershed for the development of diabetic complications indicates that hormonal factors may be important. ${ }^{1}$ Risk of MA during puberty is greater in females than males, even where $\mathrm{HbAlc}$ levels are similar. ${ }^{1}$ Perturbations in the growth hormone/IGF-I axis are thought to be important in these gender differences, but alternate mechanisms may relate to mild elevations in testosterone levels and the free androgen index that have been reported in girls with MA. ${ }^{22}$

\section{INTERVENTION}

The Diabetes Control and Complications Trial $^{23}$ showed beyond any doubt that reductions in HbAlc levels can prevent the development and progression of diabetic nephropathy and retinopathy. No threshold could be identified under which there was no risk of diabetic complications; however, considerable risk reduction can be achieved by lowering HbAlc by $1 \%$ or $2 \%$ in those with high levels (fig 1). The adolescents in the DCCT showed very similar reductions in risk to those observed in the adults, but overall HbAlc levels in both intensively and conventionally treated groups were on average $1 \%$ higher than in adults and they had a higher risk of severe hypoglytions reflect the practical problems in trying to achieve good glycaemic control in this age group.

In adults, reduction of BP to near normal levels is also effective in preventing the progression of retinopathy and nephropathy. The use of ACE inhibitors is now common in adults with MA. Use of ACE inhibitors may also have benefits in the prevention of cardiovascular disease $\mathrm{e}^{25}$ and in the development of severe retinopathy. ${ }^{26}$ caemia and weight gain. ${ }^{24}$ These observa-
There have been few studies of ACE inhibition in adolescents with TIDM, but these indicate that it may have similar effects in reversing $\mathrm{MA}^{27}$; one study suggested it may prevent the morphological changes on renal biopsy specimens associated with progression of MA. ${ }^{28}$ However, ACE inhibition can be associated with a troublesome cough in around 10\% of patients, and compliance with therapy has never been tested in the adolescent age group. Furthermore, these drugs are potentially teratogenic and unwanted pregnancy would have to be avoided. Finally, up to $30-50 \%$ of teenagers with MA may revert to normoalbuminuria at the end of puberty. ACE inhibition in subjects with transient MA may prevent renal damage during puberty and thus alleviate any later nephropathy risk, but this hypothesis has never been tested in a randomised controlled trial.

\section{SUMMARY}

Annual screening for early markers of microvascular disease during puberty should be encouraged as this is the time when they may first become evident. Regular retinal fundoscopy should be routine, but the prevalence of severe retinopathy is relatively low until the late adolescent years and further study is required to examine the cost effectiveness of introducing regular retinal photography. Clinic measurements of BP, although of value, may be less helpful than 24 hour ambulatory home BP monitoring, but this requires further evaluation. Serial assessment of albumin excretion rates or ACR in early morning urine may detect those most at risk for future nephropathy, but it is not clear whether transient MA during puberty also reflects an increased genetic risk for future complications. Where persistent MA is detected, attempts must be made to improve glycaemic control. The use of ACE inhibitors in this age group remains controversial. It should probably be limited to those subjects with proven hypertension on ambulatory BP monitoring, or a very strong family history of hypertension, type II DM, or cardiovascular disease. Although the use of ACE inhibition in cases of transient MA might provide renal protection, the efficacy, tolerance, and safety of such therapy has not yet been proven.

Arch Dis Child 2002;87:10-12

\section{Authors' affiliations}

C J Schultz, D B Dunger, R Amin, Department of Paediatrics, Addenbrooke's Hospital, Cambridge CB2 2QQ, UK

Correspondence to: Prof. D B Dunger, Department of Paediatrics, Addenbrooke's Hospital, Cambridge CB2 2QQ, UK ; david.dunger@paediatrics.ox.ac.uk

\section{REFERENCES}

1 Schultz CJ, Konopelska-Bahu T, Dalton RN, et al. Microalbuminuria prevalence varies with 
age, sex and puberty in children with insulin dependent diabetes followed in a longitudina study from diagnosis. Diabetes Care 1999;22:495-502.

2 Burger W, Hövener G, Düsterhus R, et al. Prevalence and development of retinopathy in children and adolescents with type-1 (insulin dependent) diabetes mellitus. A longitudina study. Diabetologia 1986;29:12-22.

3 Barkai L, Vamosi I, Lukacs K. Enhanced progression of urinary albumin excretion in IDDM during puberty. Diabetes Care 1998;21:1019-23.

4 Stephenson JM, Fuller JH. Microalbuminuria is not rare before 5 years of IDDM. EURODIAB IDDM Complications Study Group and the WHO Multinational Study of Vascula Disease in Diabetes Study Group. J Diabetes Complications 1994:8: 166-73.

5 Couper JJ, Clarke CF, Byrne GC, et al. Progression of borderline increases in albuminuria in adolescents with insulin-dependent diabetes mellitus. Diabet Med 1997;14:766-71.

6 D'Annuzio G, Malvezzi F, Vitali L, et al. A 3-19 year follow-up study on diabetic retinopathy in patients diagnosed in childhood and treated with conventional therapy. Diabe Med 1997; 14:951-8.

7 Kernell A, Dedorsson I, Johansson B, et al. Prevalence of diabetic retinopathy in children and adolescents with IDDM. A population-based multicentre study. Diabetologia 1997:40:307-10.

8 Rudberg S, Osterby R, Bangstad HJ, et al. Effect of angiotensin converting enzyme inhibitor or beta blocker on glomerular structural changes in young microalbuminuric patients with type I (insulin-dependent) diabetes mellitus. Diabetologia 1999:42:589-95

9 Schultz CJ, Dalton RN, Turner C, et al. Freezing method affects the concentration and variability of urine proteins and the interpretation of data on microalbuminuria. Diabet Med 2000:17:7-14.

10 Schultz CJ, Neil HAW, Dalton RN, et al. Risk of nephropathy can be detected before the onset of microalbuminuria during the early years from diagnosis of type- 1 diabetes mellitus. Diabetes Care 2000;23:1811-15.

11 Barkai L, Vamosi I, Lukacs K. Enhanced progression of urinary albumin excretion in IDDM during puberty. Diabetes Care 1998:21:1019-23.

12 Schultz CJ. The early natural history and determinants of microalbuminuria in childhood type-1 diabetes. The Oxford Regional Prospective Study of Childhood Diabetes. PhD thesis, Oxford University, 2000

13 Danne T, Weber B, Hartmann R, et al. Long-term glycaemic control has a nonlinear association to the frequency of background retinopathy in adolescents with diabetes. Diabetes Care 1994;17:1390-6.

14 Schultz CJ, Dunger DB, Neil HAW. Blood pressure does not rise before the onset of microalbuminuria in children followed from diagnosis of type-1 diabetes mellitus. Diabetes Care 2001;24:555-60.

15 Poulsen PL, Hansen KW, Mogensen CE. Ambulatory blood pressure in the transition from normo- to microalbuminuria. A longitudinal study in IDDM patients. Diabetes 1994:43: 1248-53.

16 Lloyd C, Klein R, Maser R, et al. The progression of retinopathy over 2 years: the Pittsburg epidemiology of diabetes complications (EDC) study. J Diabetes Complications 1995;9:140-8.

17 The Diabetes Control and Complications Trial Research Group. Clustering of ong-term complications in families with diabetes in the diabetes control and complications trial. Diabetes 1997;46: 1829-39.

18 Roglic G, Colhoun HM, Stevens LK, et al. Parental history of hypertension and parental history of diabetes and microvascular complications in insulin-dependent diabetes mellitus: the EURODIAB IDDM Complications Study. Diabet Med 1998;15:418-26.

19 Fagerudd JA, Pettersson FK, Gronhagen RC, et al. The impact of a family history of type II (non-insulin-dependent) diabetes mellitus on the risk of diabetic nephropathy in patients with type I (insulin-dependent) diabetes mellitus. Diabetologia 1999;42:519-26.
20 Rudberg S, Stattin EL, Dahlquist G. Familial and perinatal risk factors for micro- and macroalbuminuria in young IDDM patients. Diabetes 1998;47:1121-6.

21 Rippin JD, Patel A, Bain SC. Genetics of diabetic nephropathy. Best Pract Res Clin Endocrinol Metab 2001;15:345-58

22 Rudberg S, Persson B. Indications of low sex hormone binding globulin (SHBG) in young females with type 1 diabetes, and an independent association to microalbuminuria. Diabet Med 1995;12:816-22.

23 The Diabetes Control and Complications Trial Research Group. The effect of intensive treatment of diabetes on the development and progression of long-term complications in insulin-dependent diabetes mellitus. N Engl J Med 1993;329:977-86.

24 Diabetes Control and Complications Trial Research Group. Effect of intensive diabetes treatment on the development and progression of long-term complications in adolescents with insulin-dependent diabetes mellitus: Diabetes Control and Complications Trial. J Pediatr 1994; 125:177-88.

25 Anonymous. Effects of ramipril on cardiovascular and microavascular outcomes in people with diabetes mellitus: results of the HOPE study and MICRO-HOPE substudy. Lancet 2000;355:253-9.

26 Chaturvedi N, Sjolie AK, Stephenson JM, et al. Effect of lisinopril on progression of retinopathy in normotensive people with type 1 diabetes. The EUCLID Study Group. EURODIAB Controlled Trial of Lisinopril in Insulin-Dependent Diabetes Mellitus, Lancet 1998;351:28-31.

27 Cook J, Daneman D, Spino $M$, et al. Angiotensin converting enzyme inhibitor therapy to decrease microalbuminuria in normotensive children with insulin-dependent diabetes mellitus. J Pediatr 1990; 1 17:39-45

28 Rudberg S, Osterby R, Bangstad HJ, et al. Effect of angiotensin converting enzyme inhibitor or beta blocker on glomerular structural changes in young microalbuminuric patients with type I (insulin-dependent) diabetes mellitus. Diabetologia 1999;42:589-95.

\section{IMAGES IN PAEDIATRICS}

\section{Borrelia lymphocytoma ("lymphadenosis benigna cutis")}

A 8 year old boy had been bitten by a tick in his left axilla five months ago. A few weeks later, two erythematous macules, each $7-10 \mathrm{~cm}$ in diameter, had developed over his left chest and within a month both lesions disappeared untreated. Two months later a red-purple, firm, indolent swelling of his left nipple appeared and increased in size over the next eight weeks (see fig). He was sent to us for biopsy to rule out a tumour. Based on history we made a clinical diagnosis of Borrelia lymphocytoma, refrained from biopsy, and recommended treatment with doxycycline for four weeks. Serological assays (EIA and immunoblot) revealed specific IgG antibodies against Borrelia burgdorferi. One month later the swelling of the nipple had substantially decreased. Another four weeks later the appearance of the boy's nipple was normal. Swelling of a nipple should prompt diagnostic tests for Borrelia burgdorferi infection.

S Gissler

U Heininger

University Children's Hospital (UKBB), PO Box, CH-4005 Basel, Switzerland; Ulrich.Heininger@unibas.ch

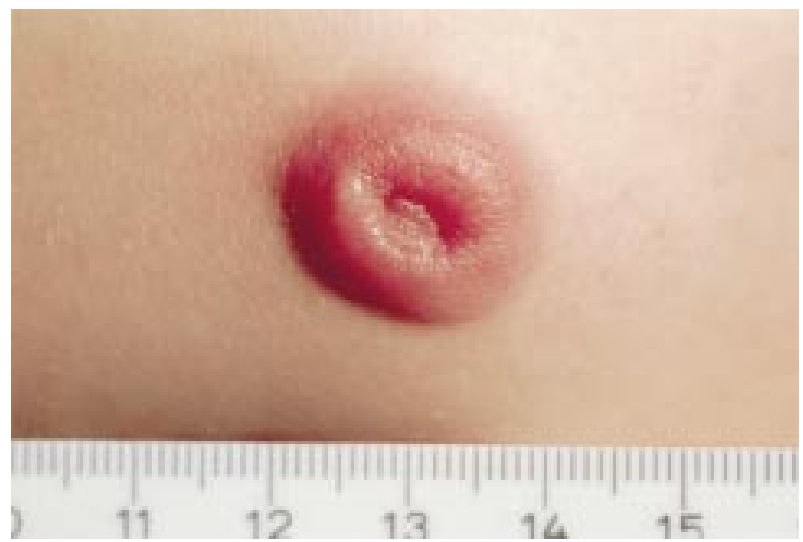

